\title{
The trigemino-cardiac reflex: a view to the future
}

Nora Sanduํㅡ, Bernhard Schaller ${ }^{2}$

${ }^{1}$ Department of Neurosurgery, University of Paris, France

${ }^{2}$ University of Oradea, Romania

Submitted: 11 August 2009

Accepted: 27 September 2009

Arch Med Sci 2010; 6, 1: 138-139

DOI 10.5114/aoms.2010.13523

Copyright ( 2010 Termedia \& Banach

After the trigemino-cardiac reflex (TCR) was first described by the senior author in 1999 [1], it resolved one of the great problems and mysteries in neurosurgery. In the meantime, several other groups have published their results about the TCR, but there still remain a lot of questions about this reflex that are currently unresolved. Our group has recently published an important update of the current knowledge of the TCR [2]. In the following, we will shed light on future research aspects of the TCR.

Of course, skull base surgery has made great advances during the last years [3], but additionally the TCR has substantially contributed to the positive development of this young surgical discipline. However, it is best known that education has a great influence on the outcome of cardiovascular events, the TCR being an example [4], so it is important that every neuroscientist knows about the TCR and its treatment, and is continuously informed about new knowledge regarding the TCR.

Despite the importance and strength of the TCR there is little information about the cellular mechanisms and brainstem pathways that constitute this reflex [5]. Stimulation of trigeminal fibres evokes a powerful excitatory and polysynaptic pathway to cardiac vagal neurons [6], and this pathway is endogenously modulated and differentially enhanced, and depressed, by serotonin (5-HT1A and 5-HT2 respectively) receptors [7]. Brainstem 5-HT systems are involved in the protective responses to hypoxia recruits a 5 -HT pathway to cardiac vagal neurons that activates 5-HT3 receptors on cardiac vagal neurons to maintain parasympathetic cardiac activity during hypoxia. The TCR is one of the known endogenous physiological neuroprotective mechanisms against ischaemia [5]. The TCR is an example of these protective physiological entities $[5,8,9]$ and may be part of a group of related responses generally defined as "oxygenconserving reflexes" [5]. Within seconds after the initiation of such a reflex, there is powerful and differentiated activation of sympathetic nerves and consequently primary cerebrovascular vasodilatation [5]. By this physiological response, adjustments of the systemic and cerebral circulations are initiated to change the cerebral blood flow in a manner that is not yet understood [5]. TCR, hibernation and ischaemic tolerance appear to involve at least partially similar physiological mechanisms [2, 9], because most of the signals, transducers and effectors that are well established in ischaemic tolerance have also been demonstrated in hypoxia tolerance or hibernating animals [10, 11]. There was a dispute over whether in humans the oxygen conservation part of the mechanism functions at all. But new research on the TCR is beginning to point in a somewhat different direction. Perhaps, when it comes to this diving adaptation, humans may be closer to whales than most researchers have realized until now. It appears that the cerebrovascular response to hypoxaemia is, to

\author{
Corresponding author: \\ Bernhard Schaller, MD, DSC, \\ DED \\ University of Oradea, \\ Romania \\ E-mail: \\ skull_base_surgery@yahoo.de
}


a large extent, due to this reflex and is generated by the activation of neurons of the rostral ventrolateral reticular nucleus; the existence of such endogenous neuroprotective strategies may extend beyond the actually known clinical appearance of the TCR [9]. Thus, 5-HT plays a critical role in the control of vagal outflow to the heart; however, why so many different receptors are involved, and their relative functional roles, remain unresolved, but further research on the TCR may also help to uncover this.

We have previously mentioned in our research that hypercapnia, hypoxaemia, and insufficient anaesthesia are predisposing factors in the occurrence of TCR [1]. In addition, the nature of the provoking stimulus, meaning its strength and duration, contributes to the significance of the TCR $[2,9]$. On the other hand, the TCR occurs more pronouncedly in children [9], attributed to the higher resting vagal tonus. It has been claimed that the TCR normally fatigues with repetitive stimuli $[2,9]$. In the near future, these facts need confirmation through further studies.

The clinical importance of the TCR lies in the fact that its clinical features range from sudden onset of sinus bradycardia, bradycardia terminating systole, asystole with no preceding bradycardia, arterial hypotension, apnoea and gastric hypermobility. Several authors have stressed the importance of avoiding the TCR during skull base surgery to improve postoperative functional outcome. In addition, the discussion about the TCR reinforces the importance of adjusting the degree of stretch or pressure to be given to trigeminal innervated structures during neurosurgery. Most cases of TCR will resolve spontaneously without any further therapeutic measures. If resolution does not happen during a reasonable amount of time after cessation of the evolving surgical manoeuvre, atropine or glycopyrrolate should be administered intravenously [2, 9].

Being familiar with the presentation, preventive measures, and management procedures is seemingly the most important aspect of the TCR. Further studies, preferably with a multicentre design, are necessary to confirm the nature, description, predisposing and triggering factors, and other aspects of this seemingly physiological phenomenon. A better and more detailed knowledge of the cascades, transmitters and molecules engaged in the TCR may also provide new insights into novel therapeutic options for a range of disorders characterized by neuronal cell death.

\section{References}

1. Schaller B, Probst R, Strebel S, Gratzl O. Trigemino-cardiac reflex during surgery in the cerebellopontine angle. J Neurosurg 1999; 90: 215-20.
2. Schaller B, Cornelius JF, Prabhakar H, et al. TrigeminoCardiac Reflex Examination Group (TCREG). The trigeminocardiac reflex: an update of the current knowledge. J Neurosurg Anesthesiol 2009; 21: 187-95.

3. Sandu N, Schaller B. Editorial: pneumocephalus after influenca virus infection and its implication to modern skull base surgery. Arch Med Sci 2008; 4: 471-3.

4. Schaller B. Eductional attainment and risk of stroke and myocardial infarction. Med Sci Monit 2003; 9: LE27.

5. Schaller B, Buchfelder M. Trigemino-cardiac reflex: a recently discovered "oxygen-conserving" response? The potential therapeutic role of a physiological reflex. Arch Med Sci 2006; 2: 3-5.

6. Gorini CJ, Jameson HS, Mendelowitz D. Serotonergic modulation oft he trigeminocardiac reflex neurotransmission to cardiac vagal neurons in the nucleus ambiguus. J Neurophysiol 2009; 102: 1443-50.

7. Schaller B, Filis A, Buchfelder M. Cardiac autonomic control in neurosurgery. Arch Med Sci 2007; 4: 287-92.

8. Schaller B. The role of endothelin in stroke: Experimental data and underlying pathophysiology. Arch Med Sci 2006; 2: 146-58.

9. Schaller B. Trigeminocardiac reflex. A clinical phenomenon or a new physiological entity? J Neurol 2004; 25: 658-65.

10. Schaller B, Cornelius JF, Sandu N, et al. Oxygen-conserving reflexes of the brain: the current molecular knowledge. J Cell Mol Med 2009; 13: 644-7.

11. Matusik E, Wajgt A, Janowska J, et al. Cell adhesion molecular markers in ischaemic stroke patients: correlation with clinical outcome and comparison with primary autoimmune disease. Arch Med Sci 2009; 5: 182-9. 